\title{
Incidence of normal micturition in myelomeningocele patients
}

\author{
I Reiner, M Jones, S Donnell, A M K Rickwood
}

\begin{abstract}
Among 108 consecutive patients with myelomeningocele aged 5-12 years initially treated by a selective policy, seven $(6.5 \%)$ achieved spontaneous urinary continence, five with normal micturition and two with urgency. All had positive conus reflexes and incomplete cord lesions which, with one exception, comprised motor as well as sensory sacral sparing.
\end{abstract}

Neuropathic bladder, with urinary incontinence and the risk of renal complications, is a major problem for patients with myelomeningocele and especially for those with smaller lesions whose handicaps otherwise tend to be slight. Reports of patients originally treated on a nonselective basis indicated normal urinary control in $23 \%^{1}$ to $33 \%^{2}$ of cases, including a few with thoracolumbar lesions. In a series of children initially managed selectively $51 \%$ were stated to be continent of urine. ${ }^{3}$ Because myelomeningocele almost always involves the terminal spinal cord, these proportions seem surprising and we considered that this issue deserved re-examination.

\section{Patients and methods}

Casenotes were reviewed of all 108 surviving myelomeningocele patients treated at the Mersey Regional Neonatal Surgical Unit during the years $1979-85$ inclusive. A selective treatment policy, employing Lorber's criteria, ${ }^{4}$ had been practised during this period except in that a few neonates with thoracolumbar lesions had been actively managed at the parents' request. Surgical closure of the spinal lesions was undertaken neonatally.

Patients whose notes indicated normal urinary control, or did not exclude this possibilty, attended for review when details of their pattern of micturition were recorded and their spinal neurology was examined.

Regional Department of Paediatric Urology, Royal Liverpool Children's Hospital (Alder Hey), Eaton Road, Liverpool L12 2AP

I Reiner

M Jones

$S$ Donnell

A M K Rickwood

Correspondence to

Mr Rickwood.

Accepted 7 January 1992

(Arch Dis Child 1992;67:640-1)

\section{Results}

One patient had been lost to follow up. Sixty five children were under the care of the urology department with a urodynamically proved neuropathic bladder and the notes of 31 others clearly indicated this diagnosis ( 15 had intermittent or indwelling urethral catheterisation, 10 had constant urinary leakage with the use of incontinence pads, five had urinary diversion, and one was dry due to regular bladder expression).
Among the 11 patients reviewed clinically, four had gross urinary and faecal incontinence. Five had an entirely normal pattern of micturition by day although one had primary nocturnal enuresis. Two children were fully continent but had marked urgency of micturition.

Neurological examination of the seven children with spontaneous daytime urinary continence showed a primary neurological level (that to which motor and sensory functions were entirely normal) ranging from L3 to S2. All had positive conus reflexes and an incomplete cord lesion which, except for one girl with urgent micturition, comprised motor as well as sensory sparing in the second to fourth sacral segments. None had suffered symptomatic urinary infections; recent ultrasound examinations or intravenous urography had shown normal upper renal tracts. Bowel habit was normal in all cases.

The incidence of spontaneous urinary continence in relation to the anatomical level of the cord lesion was thoracolumbar $0 / 8$, lumbar $3 / 44$ $(7 \%)$, lumbosacral $3 / 39(8 \%)$, and sacral $1 / 16$ $(6 \%)$.

\section{Discussion}

The incidence of spontaneous urinary continence in the present series, $6.5 \%$, is far less than that previously claimed of children treated by nonselective $^{1 / 2}$ or selective ${ }^{3}$ policies and lesser still when considering those with entirely normal daytime micturition.

Normal urinary control would be expected only of patients with positive conus reflexes and incomplete cord lesions with both motor and sensory sacral sparing, as were the findings in the present series. Incomplete cord lesions are quite common with sacral and lumbosacral myelomeningoceles but unusual with lumbar and exceptional with thoracolumbar lesions. As a rule there is only sensory sacral sparing and when this is accompanied by positive conus reflexes a precarious form of urinary control may be achieved but one which is undoubtedly abnormal. ${ }^{5}$ Urgent micturition is associated with detrusor hyperreflexia and detrusorsphincter dyssynergia; residual urine is common and upper renal tract complications may develop. A few patients with a combination of detrusor areflexia and moderate urethral resistance manage to stay more or less dry by regular voiding by abdominal straining or compression. Possibly previous reports included as having normal urinary control some patients precariously continent by these abnormal means, others where the casenotes were inaccurate 
and, perhaps, a few with pure meningoceles rather than myelomeningoceles.

Our findings have prognostic implications. Neonatally it is impossible to be certain of sacral motor sparing but conus reflexes are readily demonstrated and so also is sensory sparing by general arousal to perianal pinprick. No matter how slight the neurological deficit otherwise, neuropathic bladder is virtually certain in an infant with negative conus reflexes or a sensory complete cord lesion. By contrast, one with a combination of positive conus reflexes and a sensory incomplete cord lesion may ultimately prove to have normal bladder and bowel control.

1 Brereton RJ, Zachary RB, Lister J. Urinary continence in open myelomeningocele. Arch Dis Child 1977;52:703-7.

2 Eckstein HB. Urinary control in children with myelomeningocele. Br f Urol 1968;40:191-5.

3 Lorber J, Salfield WA. Results of selective treatment of spina bifida cystica. Arch Dis Child 1981;56:822-30.

4 Lorber J. Spina bifida cystica: results of treatment of 270 consecutive cases with criteria for selection for the future. Arch Dis Child 1972;47:854-73.

5 Rickwood AMK. The neuropathic bladder in children. In: Mundy AR, Stephenson TP, Wein AJ, eds. Urodynamics: principles, practice and applications. London: Churchill Livingstone, 1984: 326-42.

\title{
Treatment of refractory ulcerative oesophagitis with omeprazole
}

\author{
A M Dalzell, J W Searle, M K Patrick
}

\begin{abstract}
A 7 year old boy with ulcerative oesophagitis failed to respond to a two month course of intensive medical treatment using $\mathrm{H}_{2}$ receptor antagonist treatment in combination with domperidone and sucralfate. He demonstrated complete resolution using omeprazole $10 \mathrm{mg}$ once a day for eight weeks.
\end{abstract}

The aetiology of reflux oesophagitis is multifactorial. However, patients do have more frequent reflux episodes and poorer oesophageal acid clearance than normal individuals. ${ }^{1}$ Conventional medical management of oesophagitis in childhood is based on positioning and the use of pharmacological agents including $\mathrm{H}_{2}$ receptor antagonist treatment. Although the usual clinical outcome is effective resolution of symptoms, a proportion of patients with severe oesophagitis fail to respond. Omeprazole as an alternative treatment to $\mathrm{H}_{2}$ receptor antagonists has been extensively reported in adults with ulcerative oesophagitis and peptic ulcer disease, ${ }^{2}$ but not in children.

Children's Nutrition

Research Centre

The Royal Children's

Hospital Brisbane,

Herston Road, Brisbanë,

Queensland,

4029 Australia

A M Dalzell

M K Patrick

Department of

Pathology,

Royal Brisbane Hospital

J W Searle

Correspondence to:

Dr Patrick.

Accepted 14 October 1991

(Arch Dis Child 1992;67:641-2)

\section{Case report}

A 7 year old boy presented with vomiting associated with postprandial colicky abdominal pain and a weight loss of $4 \mathrm{~kg}$ in the preceding six weeks. There was a history of effortless vomiting from birth, which had not previously been investigated. Despite this, growth parameters were appropriate for age: weight 23.5 $\mathrm{kg}$ ( 50 th centile), height $119.5 \mathrm{~cm}$ ( 50 th centile), and clinical examination was unremarkable but for borborygmi and non-specific abdominal discomfort.

A blood film suggested a mild iron deficiency anaemia with a haemoglobin concentration of
$108 \mathrm{~g} / \mathrm{l}$, mean corpuscular volume $68 \mathrm{fl}$ (normal range 7-95) and mean corpuscular haemoglobin $23 \mathrm{pg}(25-30)$. Abdominal ultrasound and a barium meal and follow through were normal. Liver function tests, serum electrolyte concentrations, amylase and lipase, and urine and faeces microscopy and culture were also normal. Oesophageal manometry was not performed.

OESOPHAGEAL PH PROFILE

Oesophageal $\mathrm{pH}$ was evaluated using a $\mathrm{pH}$ sensitive antimony electrode introduced via the nares, and positioned $4 \mathrm{~cm}$ above the lower oesophageal sphincter under fluoroscopic control. Normal activity was allowed for the period of study (24 hours), and data were recorded on a solid state recorder and analysed using a computer programme (Grant Instruments (Cambridge) Ltd, Squirrel Meter/logger).

When compared with normal data taken from the work of Sondheimer and Haase, ${ }^{3}$ the results demonstrated significant and abnormal gastrooesophageal reflux. The lower oesophageal $\mathrm{pH}$ was $\mathrm{pH}<4$ for $33 \%$ of the time compared with a mean (SEM) normal of $3 \cdot 2(0 \cdot 6) \%$. There were 1.5 reflux episodes/hour compared with 0.84 $(0 \cdot 11)$, and the longest episode of $\mathrm{pH}<4$ was 64 minutes compared with $31 \cdot 6(18 \cdot 9)$.

\section{UPPER GASTROINTESTINAL ENDOSCOPY}

Upper gastrointestinal endoscopy showed circumferential ulceration and exudate in the lower third of the oesophagus. The stomach and duodenum were normal. The symptoms and oesophageal appearance did not resolve with a 12 week course of ranitidine $(75 \mathrm{mg}$ twice a day), together with domperidone (10 $\mathrm{mg}$ three times a day), and sucralfate ( $500 \mathrm{mg}$ twice a day) (fig la). Omeprazole $10 \mathrm{mg}$ once a day was 